\title{
Brain parcellation based on information theory
}

\author{
Ester Bonmati, Anton Bardera, Imma Boada \\ Institute of Informatics and Applications, University of Girona, Spain
}

\begin{abstract}
Background and objective: In computational neuroimaging, brain parcellation methods subdivide the brain into individual regions that can be used to build a network to study its structure and function. Using anatomical or functional connectivity, hierarchical clustering methods aim to offer a meaningful parcellation of the brain at each level of granularity. However, some of these methods have been only applied to small regions and strongly depend on the similarity measure used to merge regions. The aim of this work is to present a robust whole-brain hierarchical parcellation that preserves the global structure of the network.
\end{abstract}

Methods: Brain regions are modeled as a random walk on the connectome. From this model, a Markov process is derived, where the different nodes represent brain regions and in which the structure can be quantified. Functional or anatomical brain regions are clustered by using an agglomerative information bottleneck method that minimizes the overall loss of information of the structure by using mutual information as a similarity measure.

Results: The method is tested with synthetic models, structural and functional human connectomes and is compared with the classic $k$-means. Results show that the parcellated networks preserve the main properties and are consistent across subjects.

Conclusions: This work provides a new framework to study the human connectome using functional or anatomical connectivity at different levels.

Email addresses: ester.bonmati@imae.udg.edu (Ester Bonmati), bardera@imae.udg.edu (Anton Bardera), imma.boada@udg.edu (Imma Boada)

Preprint submitted to Journal of Computer Methods and Programs in BiomedicineApril 12, 2018 
Keywords: Brain parcellation, hierarchical clustering, mutual information, human brain connectome, Markov process

\section{Introduction}

The human brain contains an extraordinary network of roughly one hundred billion neurons capable of sharing and processing information efficiently. The connectome models these connections as a graph, where nodes represent

5 brain areas and edges represent structural or functional connections [1, 2]. To define the nodes, parcellation methods are used to subdivide the brain cortex into different regions according to a predefined criterion (i.e., cytoarchitecture, structure, function...).

Atlas-based parcellation methods subdivide the brain by employing a threedimensional anatomical template [3]. This template can be based on cytoarchitecture, electrophysiological observations, cortical curvature patterns [4], structural or functional connectivity profiles [5], among others. A limitation of these methods is the lack of individuality as they are based on a sample dataset as opposed to the subject.

On the other hand, connectivity-based parcellation methods subdivide the brain into spatially coherent regions of homogeneous connectivity by grouping grey-matter voxels according to the similarity of their connectivity patterns [3], obtained from diffusion magnetic resonance imaging (dMRI) or functional magnetic resonance imaging (fMRI). The more popular methods are based on the $k$-means approach $[6,7,8]$, which groups voxels into $k$ non-overlapping clusters using a similarity measure. The main issues with these methods are the definition of the number of clusters a priori and the reliance on initial random sampling, as it has been shown that iterative repetitions of the same method may lead to different results [9]. To overcome these limitations, and assuming that brain networks have hierarchical properties [10, 11], several hierarchical clustering methods that compute a parcellation at each level in the hierarchy have been proposed $[12,13,14,15]$. These methods obtain brain parcellations 
at multiple granularities without the need to define the number of clusters.

Connectivity-based methods are strongly dependent on the similarity measure used by the algorithm. Gorbach et al. [13] proposed a hierarchical method that clusters voxels using the mutual information between tractograms, and obtained promising results for specific regions of the brain. The use of mutual information as a similarity measure is, therefore, an effective solution to group voxels. However, Gorbach et al's method [13] assumes that the whole cluster can be represented by only one tractogram.

\subsection{Our approach}

In this paper, we present a hierarchical parcellation method that preserves the structure of brain network with no need to define representative tractograms. We model brain networks as a random walk on the connectome by using the 40 structural or functional connectivity matrix. From this model, we quantify the brain structure. Brain regions are clustered by applying a bottom-up hierarchical method based on the information bottleneck using a control process [16]. We evaluate the parcellation method by using synthetic, structural, and functional brain networks at different scales. The robustness of the method is tested

45 by doing multiple-subject comparisons with the resulting vector of hierarchical clusters.

\section{Method}

In this section, we propose a new method to parcellate the brain. First, we introduce a brain model based on a Markov process. Then, we describe the parcellation method, which uses the information bottleneck-based method. Additionally, we describe a measure based on mutual information which is used to perform pairwise group comparisons.

\subsection{Markov process-based brain model}

A brain network can be modeled as a graph with a pair of sets $G=(V, E)$, 
$E$ the set of $e$ edges between two nodes of $V$, that denotes their anatomical or functional connectivity. This graph can be represented by a connectivity matrix $C$ with $v \times v$ elements, where $C_{i j}$ gives the connectivity weight between node $i$ and node $j$.

In this paper, we propose to model a brain network as a Markov process $\mathbf{X}=\left\{X_{0}, X_{1}, \ldots, X_{t}, X_{t+1}, \ldots\right\}$, which represents a random walk of a particle moving from one brain region to another. From this model, we can define a probability density function $p\left(X_{t}\right)=\left\{p\left(x_{1}^{t}\right), \ldots, p\left(x_{i}^{t}\right), \ldots, p\left(x_{v}^{t}\right)\right\}$, where $p\left(x_{i}^{t}\right)$ represents the probability that a particle takes the value $x_{i}$ (i.e. the particle is in the brain region $i$ ) at state $X_{t}$ (i.e. at time step $t$ ). This particle randomly moves from a node $x_{i}$ to node $x_{j}$ according to the connectivity or probability defined in the transition probability matrix, whose elements are given by

$$
p\left(x_{j}^{t+1} \mid x_{i}^{t}\right)=\frac{C_{i j}}{C_{i}},
$$

where $C_{i j}=C_{j i}, \forall i, j$ and $C_{i}=\sum_{i} C_{i j}$ is the total weight of the edges emanating from node $x_{i}$. The transition probability $p\left(x_{j}^{t+1} \mid x_{i}^{t}\right)$ defines the probability of being in node $x_{j}$ after visiting node $x_{i}$. Note that the transition probability depends only on the current state and not on the previous ones.

The transition probabilities can be used to define the transition distribution from each node $x_{i}$, which is given by

$$
\begin{aligned}
p\left(X_{t+1} \mid x_{i}^{t}\right) & =\left\{p\left(x_{1}^{t+1} \mid x_{i}^{t}\right), \ldots, p\left(x_{j}^{t+1} \mid x_{i}^{t}\right), \ldots, p\left(x_{v}^{t+1} \mid x_{i}^{t}\right)\right\} \\
& =\left\{\frac{C_{i 1}}{C_{i}}, \ldots, \frac{C_{i j}}{C_{i}}, \ldots, \frac{C_{i v}}{C_{i}}\right\} .
\end{aligned}
$$

This distribution represents the overall probability of a particle to be in a different node after visiting node $x_{i}$.

The probability of being in node $x_{i}$ can be defined by a stationary distribution [17]. In this case, for undirected brain networks, the stationary distribution is given by

$$
p\left(x_{i}\right)=\frac{C_{i}}{C_{T}}
$$

where $C_{T}=\sum_{i} \sum_{j} C_{i j}$ is twice the sum of the weights of all the edges [17]. so The stationary distribution, $p\left(X_{t}\right)=\left\{p\left(x_{1}\right), \ldots, p\left(x_{i}\right), \ldots, p\left(x_{v}\right)\right\}$, defines the 
probability of a particle to be in each of the nodes. Note that the stationary distribution of a node is proportional to the total weight of the edges emanating from that node.

\subsection{Mutual information as a measure of brain structure}

Mutual information (MI) is a well-known measure that quantifies the shared information between two different variables $X$ and $Y$ defined as

$$
\begin{aligned}
I(X ; Y) & =H(X)-H(X \mid Y) \\
& =\sum_{x \in \mathcal{X}} \sum_{y \in \mathcal{Y}} p(x, y) \log \frac{p(x, y)}{p(x) p(y)},
\end{aligned}
$$

where $H(X)=-\sum_{x \in \mathcal{X}} p(x) \log p(x)$ is the Shannon entropy of $X$ and measures the uncertainty of the variable $X$, and $H(X \mid Y)=-\sum_{y \in \mathcal{Y}} p(y) \sum_{x \in \mathcal{X}} p(x \mid y) \log p(x \mid y)$ is the conditional entropy and measures the average uncertainty associated with

90 $X$ if we know the outcome of $Y$. In our approach, we use the MI measure to quantify the shared information or similarity between two states of a Markov chain, i.e. $I\left(X_{t} ; X_{t+1}\right)$.

For a stationary Markov chain, the MI between consecutive states, $I\left(X_{t} ; X_{t+1}\right)$, coincides with the excess entropy $[18,19]$, which is a measure of system structure. We use this measure to quantify the structure of the networks. High values of MI will indicate that there is a high correlation between consecutive states and, therefore, that the brain is highly structured. Mutual information can also be seen as the difference between the uncertainty of the states without any prior knowledge and the uncertainty of the states when the past is known (or information gained when the previous node is known). Therefore, the higher the MI, the less random the connections.

\subsection{Parcellation method}

The goal of our parcellation method is to cluster the brain regions, represented as different states of a Markov chain, by minimizing the loss of information when two regions are merged, the effect of which is to maintain the 
overall structure. The agglomerative information bottleneck method, proposed by Thisby et al. [20], clusters a random variable $X$ depending on a random variable $Y$ by minimizing the loss of mutual information. Gorbach et al. [13] used this method to preserve the maximum information between the representative tractogram and all the tractograms that belonged to the same cluster. However, in our brain model, we want to preserve the maximum information between brain regions, represented as consecutive states $\left(X_{t}\right.$ and $\left.X_{t+1}\right)$ of a Markov process $\mathbf{X}$ instead of two different variables $X$ and $Y$. In this case, when two nodes of the Markov process are merged, both $X_{t}$ and $X_{t+1}$ are modified. Due to this limitation, the classic agglomerative information bottleneck method cannot be used. Thus, we use the extended version of the algorithm presented in [16], which takes this fact into consideration and enables to cluster a random variable $X$ depending on a Markov process.

The method starts by assigning each node (or brain region) to a different cluster, with a total of $v$ clusters. In the first step, the loss of mutual information due to a possible merge of every pair of nodes $\left(x_{i}, x_{j}\right)$ is calculated. The MI loss is computed as the difference of mutual information between two consecutive states $I\left(X_{t} ; X_{t+1}\right)$, when nodes $x_{i}$ and $x_{j}$ belong to different clusters, and the mutual information $I\left(\widehat{X}_{t} ; \widehat{X}_{t+1}\right)$ when nodes $x_{i}$ and $x_{j}$ have been merged into ${ }_{125} \quad$ a single node $\hat{x}[16]$ as follows

$$
\begin{aligned}
\delta I_{\widehat{i j}}(\mathbf{X}) & =I\left(X_{t} ; X_{t+1}\right)-I\left(\widehat{X}_{t} ; \widehat{X}_{t+1}\right) \\
& =2 p(\hat{x}) J S\left(x_{i}, x_{j}\right)-p(\hat{x}, \hat{x}) I\left(\widetilde{X}_{t} ; \widetilde{X}_{t+1}\right),
\end{aligned}
$$

where $p(\hat{x})=p\left(x_{i}\right)+p\left(x_{j}\right), p(\hat{x}, \hat{x})=\sum_{m=i, j} \sum_{n=i, j} p\left(x_{m}, x_{n}\right)$, and $J S\left(x_{i}, x_{j}\right)$ is the Jensen-Shannon divergence [21] between $p\left(X_{t+1} \mid x_{i}^{t}\right)$ and $p\left(X_{t+1} \mid x_{j}^{t}\right)$, which is defined as

$J S\left(x_{i}, x_{j}\right)=H\left(p\left(X_{t+1} \mid \hat{x}^{t}\right)\right)-\frac{p\left(x_{i}\right)}{p(\hat{x})} H\left(p\left(X_{t+1} \mid x_{i}^{t}\right)\right)-\frac{p\left(x_{j}\right)}{p(\hat{x})} H\left(p\left(X_{t+1} \mid x_{j}^{t}\right)\right)$,

where $p\left(X_{t+1} \mid \hat{x}^{t}\right)=\sum_{k=i, j} \frac{p\left(x_{k}\right)}{p(\hat{x})} p\left(X_{t+1} \mid x_{k}^{t}\right)$. 
The term $I\left(\widetilde{X}_{t} ; \widetilde{X}_{t+1}\right)$ is given by

$$
I\left(\widetilde{X}_{t} ; \widetilde{X}_{t+1}\right)=\sum_{m=i, j} \sum_{n=i, j} p\left(\tilde{x}_{m}, \tilde{x}_{n}\right) \log \frac{p\left(\tilde{x}_{m}, \tilde{x}_{n}\right)}{p\left(\tilde{x}_{m}\right) p\left(\tilde{x}_{n}\right)},
$$

where $p\left(\tilde{x}_{m}, \tilde{x}_{n}\right)=\frac{p\left(x_{m}, x_{n}\right)}{p(\hat{x}, \hat{x})}, p\left(\tilde{x}_{m}\right)=\frac{\sum_{n=i, j} p\left(x_{m}, x_{n}\right)}{p(\hat{x}, \hat{x})}$, and $p\left(\tilde{x}_{n}\right)=\frac{\sum_{m=i, j} p\left(x_{m}, x_{n}\right)}{p(\hat{x}, \hat{x})}$.

Next, the method finds and clusters the pair of nodes $\left(x_{i}, x_{j}\right)$ with a minimum loss of information $\delta I_{\widehat{i j}}(\mathbf{X})$. In other words, it merges the states that preserve the maximum information of the whole network.

After each merge, the MI loss of the new cluster $\hat{x}$ and any other cluster has to be calculated. This is performed using Equation 5. Note that, when two nodes are merged, the connectivity probability function $p\left(X_{t+1} \mid x_{k}^{t}\right)$ of each node $x_{k}$ changes, since the two nodes $\left(x_{i}, x_{j}\right)$ become a single node $\hat{x}$. Due to this fact, the MI loss of the other states has to be recomputed to take into account the information lost by the new cluster. The merge of nodes $\left(x_{i}, x_{j}\right)$ leads to a variation of the MI loss of nodes $\left(x_{k}, x_{l}\right)$. As shown in [16], this variation is given by

$$
\Delta \delta I_{\widehat{k l}}^{\widehat{i j}}(\widehat{\mathbf{X}})=-2 p\left(\hat{x}_{\widehat{k l}}, \hat{x}_{\widehat{i j}}\right) I\left(\widetilde{X}_{t}^{\widehat{k l}} ; \widetilde{X}_{t+1}^{\widehat{i j}}\right)
$$

where $p\left(\hat{x}_{\widehat{k l}}, \hat{x}_{\widehat{i j}}\right)=\sum_{m=k, l} \sum_{n=i, j} p(m, n)$, and $I\left(\widetilde{X}_{t}^{\widehat{k l}} ; \widetilde{X}_{t+1}^{\widehat{i j}}\right)$ is defined as

$$
I\left(\widetilde{X}_{t}^{\widehat{k l}} ; \widetilde{X}_{t+1}^{\widehat{i j}}\right)=\sum_{m=k, l} \sum_{n=i, j} p\left(\tilde{x}_{m}, \tilde{x}_{n}\right) \log \frac{p\left(\tilde{x}_{m}, \tilde{x}_{n}\right)}{p\left(\tilde{x}_{m}\right) p\left(\tilde{x}_{n}\right)} .
$$

Note that, it is much more efficient to compute Equation 8 than Equation 5. As a result, a new matrix of MI loss is obtained. The algorithm repeats the described process until all the regions have been clustered. Fig. 1 shows a graphical diagram of the main steps of the method.

\subsection{Group comparison}

An important desirable property of clustering algorithms is the consistency of results across subjects. To quantify the similarity of different parcellations, we use the normalized mutual information (NMI) [22] which has been used in similar studies [23]. NMI calculates the shared information between a pair of 


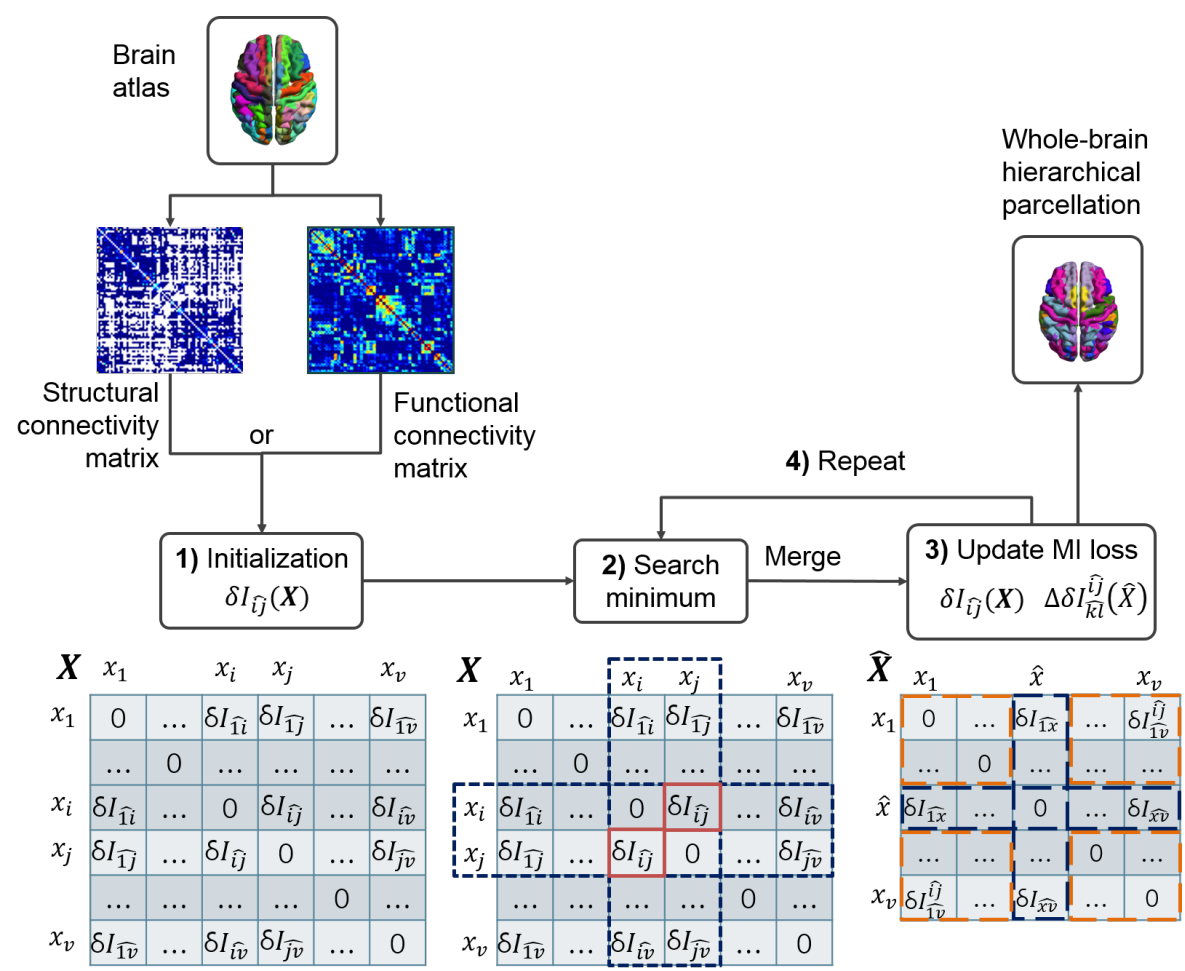

Figure 1: Diagram showing the main steps of the method. The input is a low-scale segmentation of the brain which is modeled as a Markov process. First, the loss of information for each possible pair of regions is calculated. Then, the method searches for the minimum value and the corresponding regions are merged. The loss of information is updated and the search is repeated. As a result, a whole-brain hierarchical parcellation is obtained.

clusterings and can be seen as a distance value of results obtained from different subjects. The NMI of two discrete random variables $A$ and $B$ can be defined as

$$
N M I(A, B)=\frac{I(A ; B)}{\sqrt{H(A) H(B)}} .
$$

The measure takes values between 0 and 1 , taking the value 0 if and only if $A$ and $B$ are independent. In our case, $A$ and $B$ are vectors of regions corresponding to two different results of the method, where each value corresponds to the index of the cluster that the region has been assigned. The comparison is done for a particular number of clusters. Note that, $A$ and $B$ refer to two different brain parcellations, and thus, $I(A ; B)$ is not related to the measure 
used by the proposed parcellation method.

\section{Materials}

In this section, the datasets used to perform the experiments are described in detail. We used synthetic model networks with small-world properties to test the properties of the parcellated brain networks. In addition, we analyzed human structural and functional datasets to characterize the clustered connectivity patterns and evaluate the consistency of the method across subjects.

\subsection{Synthetic modular small-world networks}

Brain networks have been shown to exhibit small-world properties [24, 25, 26, 27, 28]. We used the Brain Connectivity Toolbox (BCT) [29] to generate binary directed networks with small-world properties employing the function makeeven_CIJ. A set of 40 networks with 2048 nodes, 700,000 edges and 8 modules and a second set of 40 networks with 1024 nodes, 287,000 edges and 7 modules were created. A random weight was assigned to each edge of the network. The weighted undirected networks were obtained by copying the elements above the diagonal to below the diagonal.

\subsection{Structural connectome}

As structural networks we used the normalized whole-brain structural connection matrices created from dMRI tractography at 5 different scales [30], corresponding to 83,129, 234, 463 and 1015 cortical and subcortical regions of interest. These matrices were created from 10 subjects, all males aged $22 \pm 1.3$ years old. Edge weights were calculated by dividing the number of fibers within a region by the average of the region surface and by the average length of the fibers. The average matrices were calculated from the 10 subjects for each scale.

\subsection{Functional connectome}

The functional connectivity network used in this work was generated using the Automated Anatomical Labeling (AAL) atlas [31] which contains the cerebrum divided into 45 regions for each hemisphere. This atlas has been used 
in recent studies such as $[32,33,34]$ and it is included in the BrainNet viewer

190

(version 1.52) [35]. The surface volume template was from the ICBM152 (International Consortium from Brain Mapping). Data were created from 198 healthy subjects (76 males and 122 females), aging from 18 to 26 years old. The functional matrices were built by first parcellating the brain with the AAL atlas and the mean fMRI time courses were calculated for each pair of nodes using Pearson's correlation coefficients [35].

\section{Results and discussion}

To evaluate how the proposed method preserves the overall properties of the networks, we used standard well-known global (one value per network) and local (one value per node) network measures. Additionally, we calculated the mutual information of the networks as described in Section 2.2 to quantify the structure. As standard global measures, we calculated the density, which is defined as the fraction of connections of the nodes versus all possible connections, the assortativity coefficient, which measures if nodes highly connected tend to be connected with nodes also highly connected, the modularity, which is defined as the strength of division in which the network can be subdivided into nonoverlapping modules, and the small-world index, which tests if the network has small-world properties by considering the clustering coefficients and the characteristic path lengths. As standard local measures, we calculated the degree, which is defined as the number of links connected to the node, the strength, which is the sum of the weights, and the clustering coefficient, which is defined as the fraction of neighbors of a node that are also connected to each other. Standard measures were calculated using the BCT toolbox. For comparison purposes, we provide the results obtained employing the classic $k$-means method

by clustering the network using the correlation between brain regions (distance measure $=1$-joint probability). We tested the robustness of the method by comparing the results across subjects. 


\subsection{Synthetic modular small-world networks}

In the first experiment, we considered the 40 synthetic networks with smallworld properties and 2048 nodes created with the BCT toolbox. We applied the classic $k$-means algorithm and the proposed method to obtain 40 clustered networks with 1024 nodes. We compared the results with the original networks and also with the second set of 40 synthetic networks with 1024 nodes, which we used as a reference. Results are shown in Table 1.

Table 1: Values of standard measures for the 40 synthetic model networks with 2048 and 1024 nodes compared to the values of the clustered networks with 1024 nodes obtained by using the classic $k$-means algorithm and the proposed method (mean \pm STD)

\begin{tabular}{lllll}
\hline Measure & Original 2048 & Original 1024 & $k$-means & Clustered 1024 \\
\hline MI & $2.86 \pm 9.54 \times 10^{-4}$ & $2.14 \pm 0.002$ & $2.24 \pm 0.002$ & $2.27 \pm 0.002$ \\
Density & $0.17 \pm 9.91 \times 10^{-5}$ & $0.27 \pm 3.35 \times 10^{-4}$ & $0.27 \pm 3.93 \times 10^{-4}$ & $0.27 \pm 3.67 \times 10^{-4}$ \\
Assortativity & $0.003 \times \pm 0.002$ & $1.38 \times 10^{-4} \pm 0.002$ & $0.02 \pm 0.007$ & $5.43 \times 10^{-4} \pm 0.002$ \\
Modularity & $0.61 \pm 0.028$ & $0.32 \pm 0.013$ & $0.58 \pm 0.043$ & $0.62 \pm 0.007$ \\
Small-world & $2.97 \pm 0.005$ & $1.16 \pm 0.003$ & $1.47 \pm 0.004$ & $1.53 \pm 0.004$ \\
\hline Degree & $341.81 \pm 9.05$ & $280.85 \pm 11.29$ & $283.88 \pm 39.47$ & $281.09 \pm 14.41$ \\
Strength & $170.94 \pm 7.01$ & $140.41 \pm 7.43$ & $340.99 \pm 89.95$ & $341.01 \pm 20.61$ \\
Clustering & $0.29 \pm 0.013$ & $0.19 \pm 0.006$ & $0.59 \pm 0.089$ & $0.57 \pm 0.03$ \\
coefficient & & & & \\
\hline
\end{tabular}

As expected, the MI was higher with our approach, as it was optimized by the method. Although the results obtained were similar to the original network with 2048 nodes, some differences could be appreciated. For instance, the density was slightly higher, and the small-world index and the degree were slightly lower, due to the fact that, after clustering, connections within the same cluster were not counted. However, these measures were similar to the original network with 1024 nodes. On the other hand, the clustered networks had a higher strength because it was preserved when two nodes were clustered. Interesting properties were found with the modularity and the assortativity measures. While the modularity with our method was similar to the original network, it 
was slightly lower using $k$-means. Additionally, while the assortativity was sim-

ilar to the original network with 1024 nodes using our method, it was higher with $k$-means. These values showed that while the $k$-means failed in preserving these characteristics, our method was able to provide a network with more similar values. The clustering coefficient was higher using both $k$-means and our method, probably due to the increase in number of edges per node.

\subsection{Structural connectome}

In the following experiment we used the average structural connectome from the 10 subjects. We applied our method to the group-average connectivity matrix with 1015 partitions to obtain a clustered network of 83 regions, which we compared with the corresponding anatomical parcellation of 83 regions and the results of the $k$-means method. Results are shown in Table 2. In this case, as in the synthetic model networks, similar values were achieved for the anatomical parcellation and the clustered parcellation, which indicates that the main properties of the network were preserved. As expected, the mutual information value was higher, since this is the aim of the method. The assortativity measure, which is used to measure if nodes are connected to nodes with a similar connectivity pattern, was similar with our method but not with $k$-means, which indicates that the $k$-means network was more random.

In order to further evaluate the resulting brain parcellations, the $3 \mathrm{D}$ volumes of the group average anatomical parcellation with 1015 regions and with 83 regions are shown in Fig. 2(a) and Fig. 2(b), respectively. The results obtained with the proposed method by clustering the original network with 1015 regions into 83 regions are shown in Fig. 2(c). Differences in the generated parcellations could be appreciated. The network obtained with the proposed approach had higher mutual information than the anatomical parcellation, therefore the resulting networks provided more information about the structure. It is worth mentioning that regions tended to cluster with neighboring regions, although no spatial restrictions were used in the proposed method. A good clinical study to investigate such differences at various levels is required, which is out of the scope 
Table 2: Values of the standard network measures for the anatomical parcellation compared to values obtained from the clustered network using our method and $k$-means algorithm. All networks have a total of 83 nodes. For local measures the mean \pm standard deviation is given.

\begin{tabular}{llll}
\hline Measure & Anatomical 83 & $k$-means 83 & Clustered 83 \\
\hline MI & 2.53 & 2.71 & 3.16 \\
Density & 0.57 & 0.57 & 0.62 \\
Assortativity & -0.037 & 0.03 & -0.036 \\
Modularity & 0.45 & 0.49 & 0.56 \\
Small-world & 0.40 & 0.42 & 0.32 \\
\hline Degree & $47.13 \pm 15.15$ & $46.38 \pm 12.91$ & $50.96 \pm 12.02$ \\
Strength & $0.01 \pm 0.001$ & $0.01 \pm 0.003$ & $0.01 \pm 0.002$ \\
Clustering & $2.44 \times 10^{-4} \pm 6.95 \times 10^{-5}$ & $1.16 \times 10^{-4} \pm 3.52 \times 10^{-5}$ & $1.25 \times 10^{-4} \pm 2.49 \times 10^{-5}$ \\
coefficient & & & \\
\hline
\end{tabular}

of this work. However, with these results, we show that the proposed method may provide useful parcellations to study properties of the network such as the overall structure at different levels.

Additionally, we provide the dendrogram corresponding to the hierarchical clustering of the structural connectome with 83 regions in Fig. 3. As an example, we show the connectogram of the last four regions at the bottom of the dendrogram, corresponding to the right hemisphere banks superior temporal (Fig. 4(a)), right hemisphere transverse temporal (Fig. 4(b)), right hemisphere superior temporal (Fig. 5(a)) and the right hemisphere middle temporal (Fig. 5(b)). Due to the high density of the nodes, the connectograms only show the connections with values higher than $10^{-3}$. As it can be seen in the dendrogram of Fig. 3, the first step of the method merges the banks of the superior temporal sulcus of the right hemisphere with the right hemisphere transverse temporal gyrus. As it can be seen in Fig. 4(a) and (b), both are connected to the right hemisphere superior temporal gyrus and the banks of the superior temporal sulcus does not have strong connections. Thus, the merge of these two regions provides the minimum loss of mutual information. These two clustered regions are latter merged with the right hemisphere superior temporal gyrus 

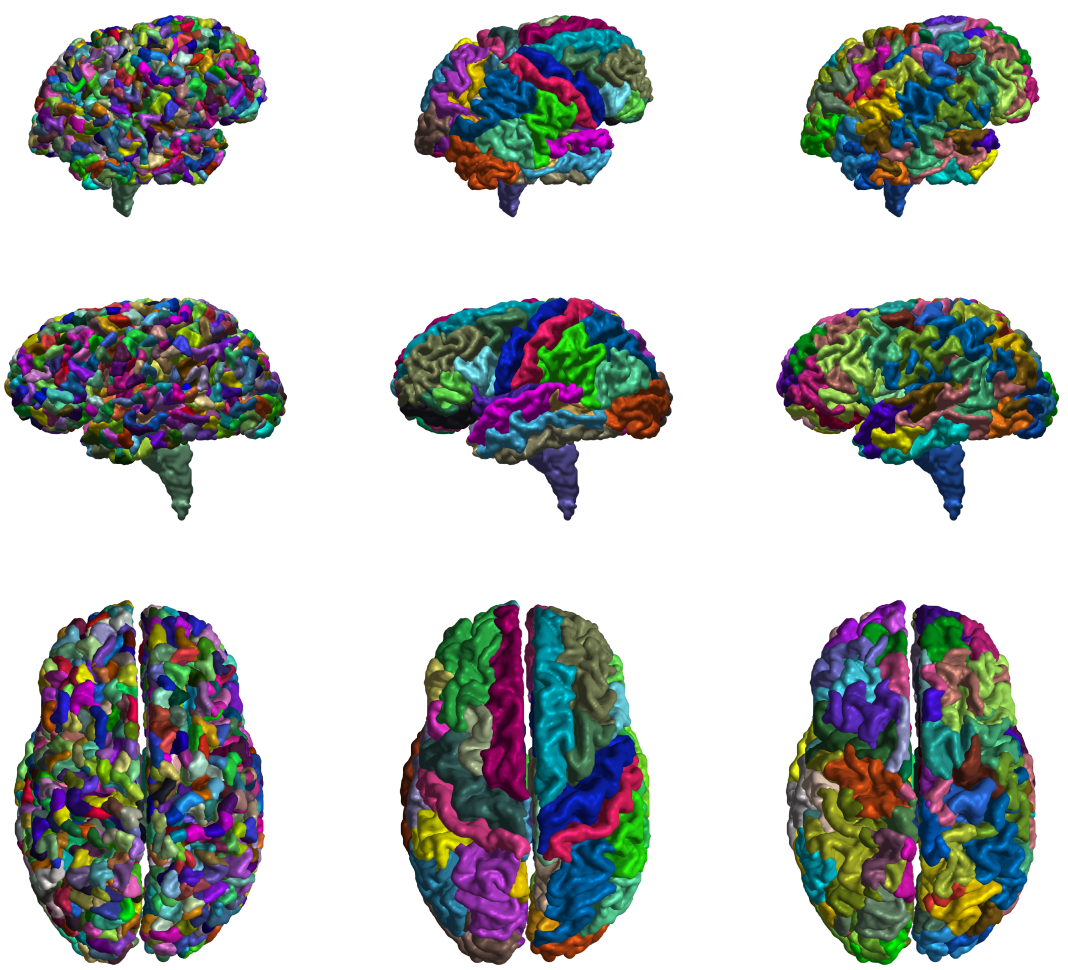

(a) Anatomical

$N=1015$

(b) Anatomical

$N=83$ (c) Clustered

$N=83$

Figure 2: (a) Anatomical parcellation with 1015 regions; (b) Anatomical parcellation with 83 regions; (c) Clustered parcellation obtained by clustering (a) into 83 regions.

and the right hemisphere middle temporal gyrus. These three regions have a very similar connectivity pattern (see Fig. 4(a) and Fig. 5(a) and (b)), hence, merging these regions the network is simplified, as one region can predict the other, thus, the loss of information is minimum and the structure is preserved.

\subsection{Functional connectome}

In the last experiment, we show the applicability of the proposed method to functional data. In this case, we used the functional matrix with 90 partitions corresponding to the AAL atlas described in Section 3.3. Fig. 6, which was 


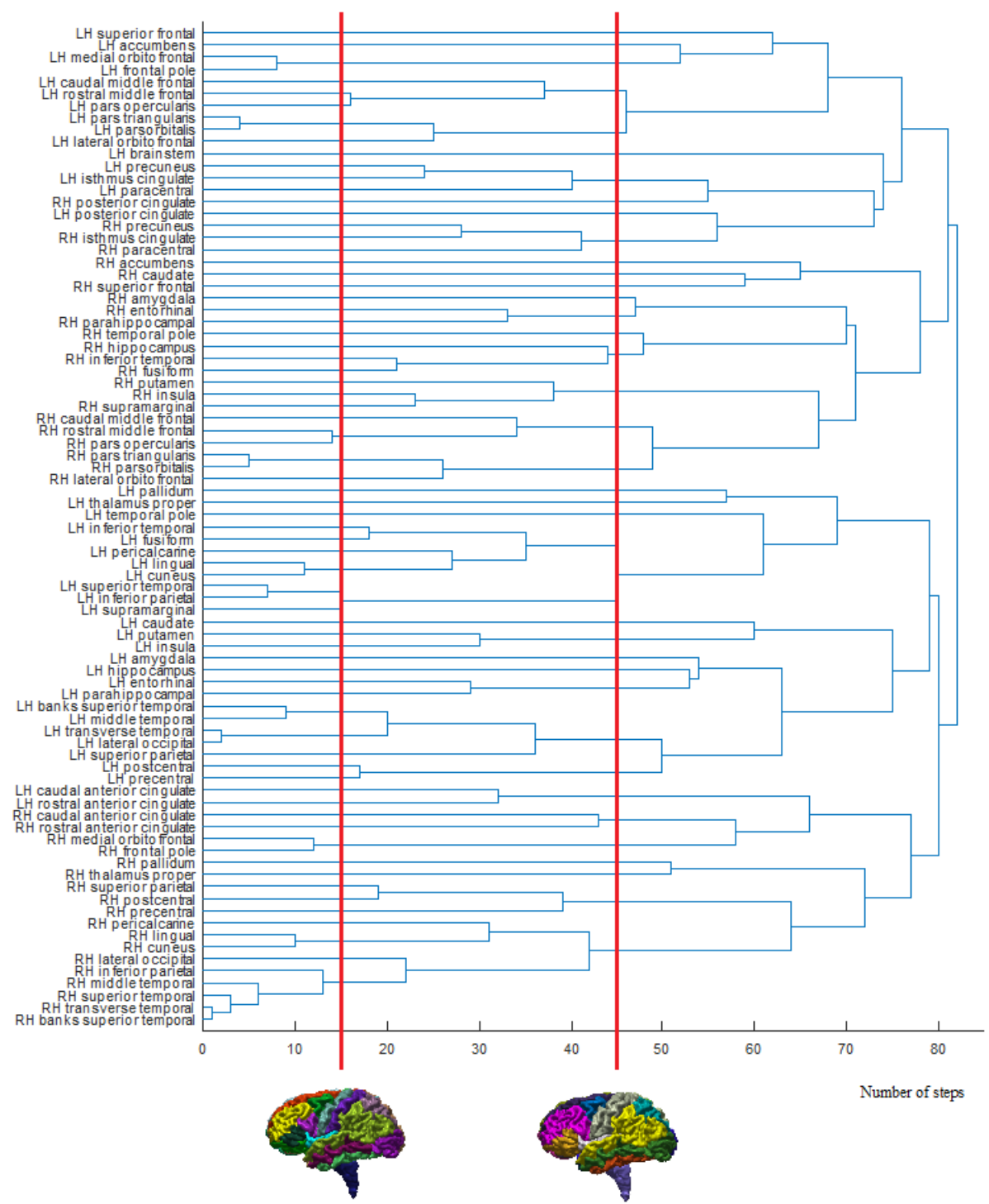

Figure 3: Dendrogram of the hierarchical clustering parcellation obtained from an anatomical segmentation with 83 regions. The two volumes at the bottom correspond to the parcellations obtained after 15 and 45 steps, respectively.

290

generated with BrainNet [35], shows the resulting volumes along with the connectivity matrix, after applying the clustering. As can be seen in the images, the method tended to join the same area from the left and the right hemi- 


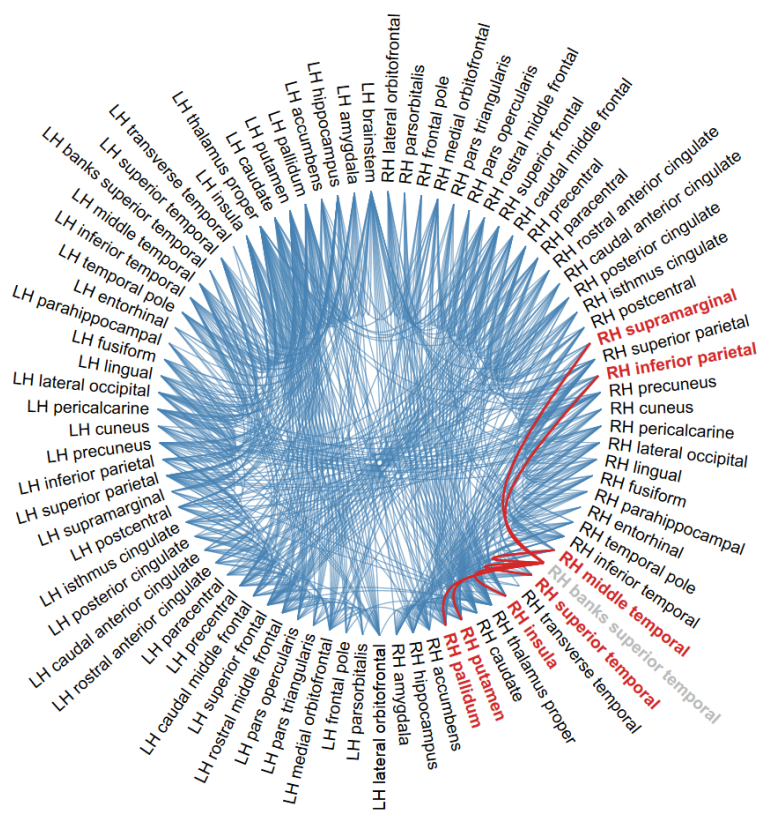

(a)

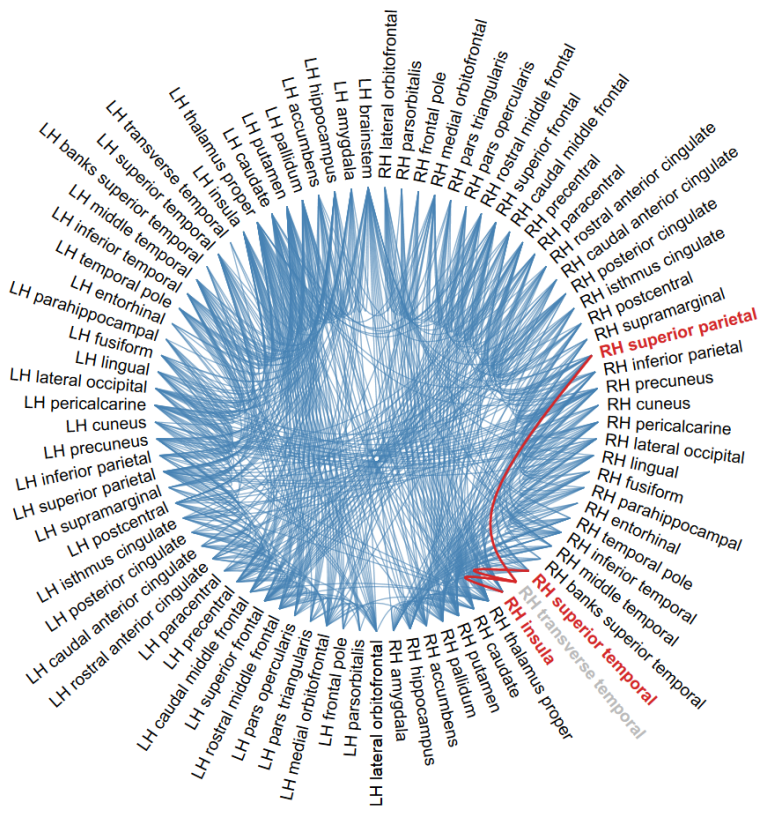

(b)

Figure 4: Connectogram of the original structural network with 83 anatomical partitions. In red, the connections of (a) the right hemisphere banks superior temporal and (b) the right hemisphere transverse temporal. 


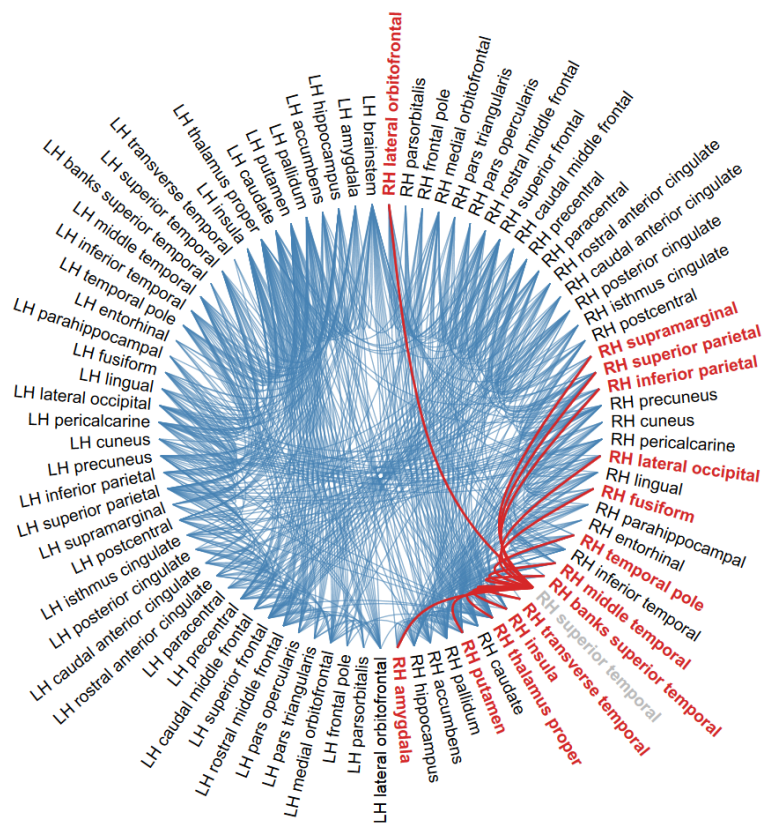

(a)

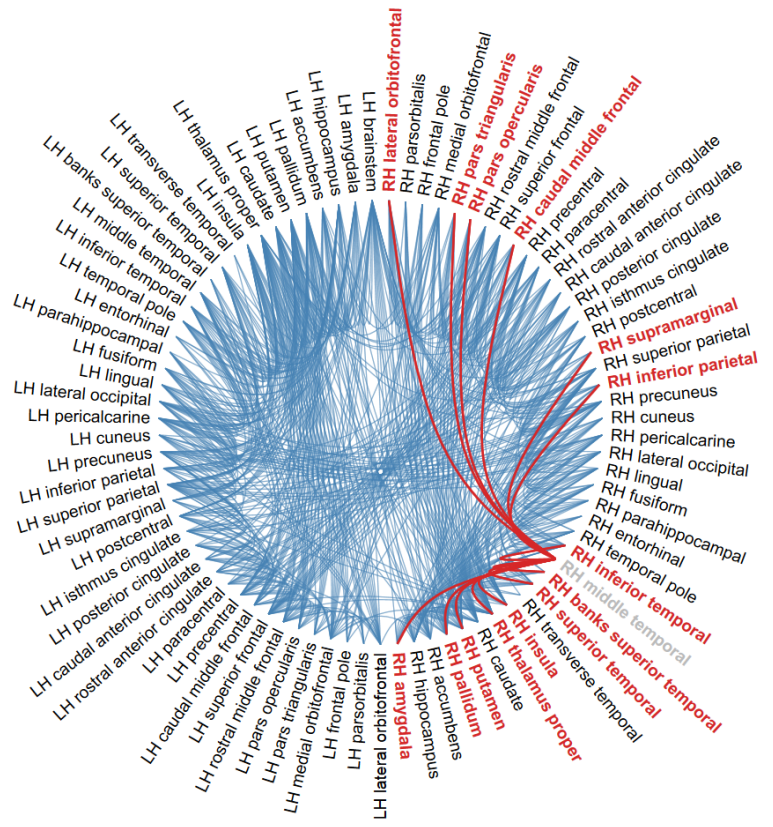

(b)

Figure 5: Connectogram of the original structural network with 83 anatomical partitions. In red, the connections of (a) the right hemisphere superior temporal and (b) the right hemisphere middle temporal. 
sphere. This was due to the similarity of the connection pattern in both areas. Thus, joining both regions the functionality of the whole-brain remained similar while the network was summarized. For a lower number of clusters, the method tended to merge areas belonging to the same lobe. As can be seen in the matrices shown in Fig. 6, the general structure of the network was preserved for all the cases.

\subsection{Group comparison}

To evaluate the consistency of the results, Fig. 7 shows the average NMI values and standard deviation of the pairwise comparison between the different parcellations obtained from the structural connectome of 10 subjects. Initially, there is a one-to-one correspondence between the parcellations, therefore NMI $=1$. Then, due to accumulated error, the NMI slowly degrades in each step of the method, as each subject region becomes clustered in a slightly different order. As expected, when the number of clusters is small, the NMI degrades quicker, having more random behavior and a greater accumulated error. As it can be seen from the NMI values in Fig. 7, the results are consistent across subjects, with less variability when the number of clusters is high.

310

Although it is still unclear which level gives the best representation, this measure can be used to provide a threshold for the minimum number of regions to consider when taking into account the desired similarity across subjects. For example, if a NMI of 0.8 is preferred, only parcellations with a minimum of 300 regions should be considered.

\subsection{Final discussion}

In this paper, we show a proof of principle demonstration of a new parcellation method that provides robust and reproducible results using dMRI and fMRI data. Although a further investigation with clinical data is required, the proposed approach provides a new framework which may be of interest in 320 studying the structure and function of complex brain networks. 

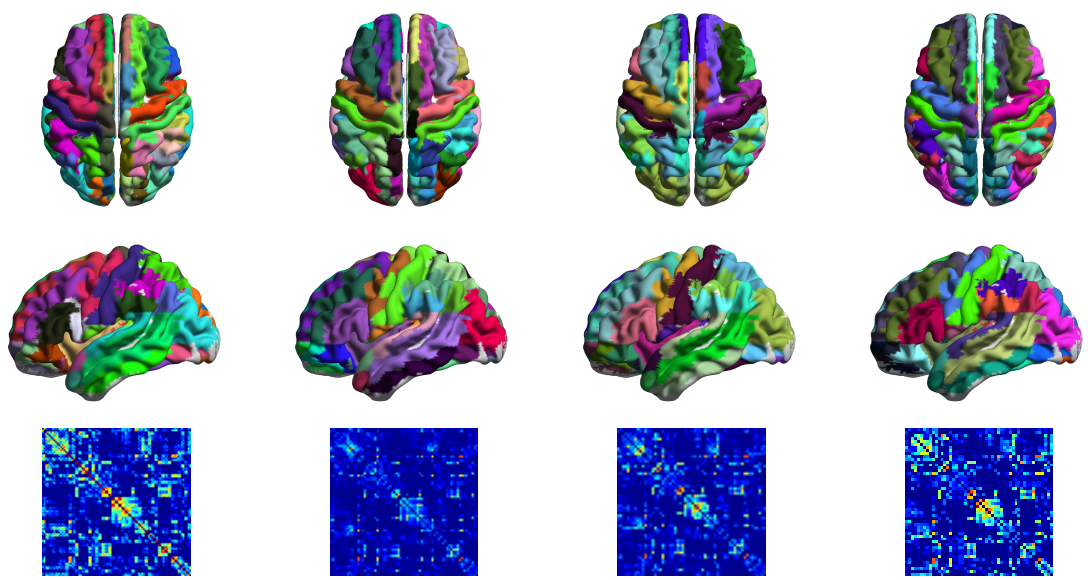

Original

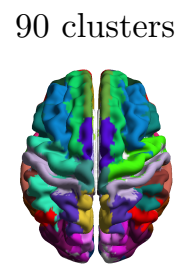

70 clusters

60 clusters

50 clusters
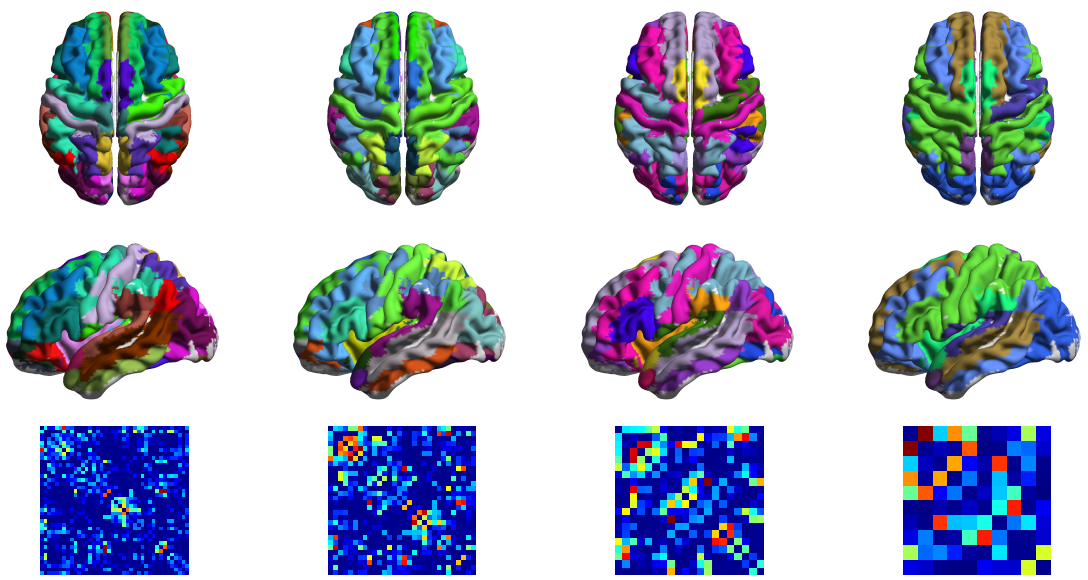

40 clusters

30 clusters
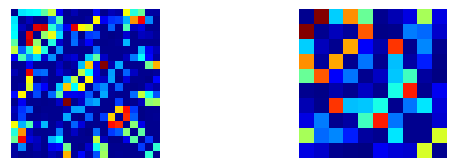

20 clusters

10 clusters

Figure 6: Illustrative example of the results obtained with the functional connectome. The method takes as input the original network with 90 clusters. This figure shows the results for $70,60,50,40,3020$ and 10 clusters.

Hierarchical clustering methods were proposed to overcome some of the limitations of connectivity-based methods such as the $k$-means algorithm, by merging only one region at each step, resulting in a tree structure. As we have shown, the proposed method leads to clustered networks with similar network metrics to the original networks but with a higher MI. We have seen that our method 


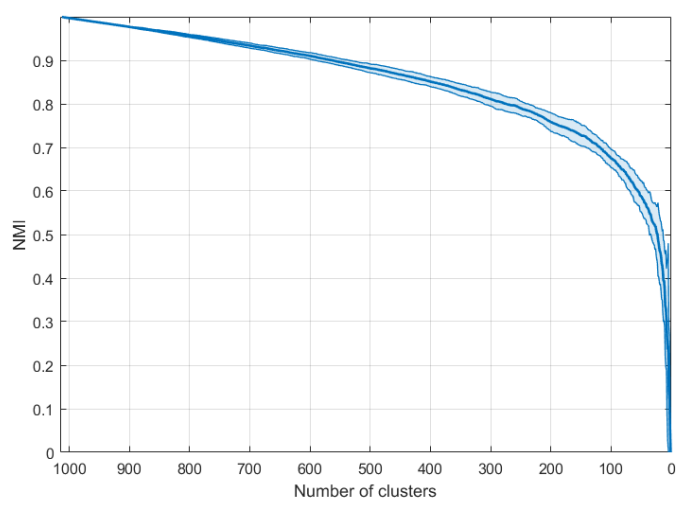

Figure 7: All possible NMI pairwise comparison for each level between the pacellations obtained from 10 subjects (mean value \pm standard deviation).

performs better than the $k$-means method, with the advantage of a hierarchical solution. One limitation of our method is that it does not guarantee a global optimum, as the proposed greedy algorithm only takes into account the local loss of information. The information bottleneck method has been used before to parcellate the brain. Gorbach et al. [13] presented an information-based parcellation method able to cluster brain regions with promising results. However, Gorbach's method assumes that there is a prototype that represents the whole cluster and adds a new parameter to control the sensitivity of the uncertainty which may affect the result of the method. In this work, by using an extended version of the bottleneck method, we eliminate the need to define a representative tractogram that defines the connectivity pattern of the cluster and no extra parameters are used.

\section{Conclusions}

In this paper, we describe a robust whole-brain hierarchical parcellation method that preserves the global structure of the network. A Markov model is defined by modeling a brain network as a random walk on the connectome. Using this model, the mutual information is used to quantify the structure of a 
network. Brain regions are clustered by employing the information bottleneck method which minimizes the loss of information between connectivity profiles when two regions are merged into the same cluster. The method is tested on synthetic model network with small-world properties, structural and functional human connectomes. We use the normalized mutual information to compare the results at different scales. From our tests, we show that the method is able to create summarized brain networks that preserve the brain structure and are consistent across subjects. With this work, we provide a new approach that could improve the understanding of the human connectome structure. Future work should focus on the variability of the results using different anatomical atlases for initialization and also include cases with large-scale lesions. A multimodal version of the method is being studied.

\section{Acknowledgments}

This work was supported by the Catalan Government (Grant No. 2014-SGR1232) and by the Spanish Government (Grant No. TIN2013-47276-C6-1-R ).

\section{References}

[1] P. Hagmann, From diffusion MRI to brain connectomics, Ph.D. thesis, EPFL, Lausanne, 2005.

[2] O. Sporns, G. Tononi, R. Kötter, The human connectome: a structural description of the human brain, PLoS Comput. Biol. 1 (2005).

[3] M.A. de Reus, M.P. van den Heuvel, The parcellation-based connectome: limitations and extensions, Neuroimage 80 (2013) 397-404.

365 [4] R.S. Desikan, F. Ségonne, B. Fischl, B.T. Quinn, B.C. Dickerson, D. Blacker, R.L. Buckner, A.M. Dale, R.P. Maguire, B.T. Hyman, M.S. Albert, R.J. Killiany, An automated labeling system for subdividing the human cerebral cortex on MRI scans into gyral based regions of interest, Neuroimage 31 (2006) 968-80. 
[5] R.C. Craddock, G.A. James, P.E. Holtzheimer, X.P. Hu, H.S. Mayberg, A whole brain fMRI atlas generated via spatially constrained spectral clustering, Hum. Brain Mapp. 33 (2012) 1914-1928.

[6] A. Anwander, M. Tittgemeyer, D.Y. von Cramon, A.D. Friederici, T.R. Knösche, Connectivity-based parcellation of Broca's area, Cereb. cortex 17 (2007) 816-25.

[7] J.C. Klein, T.E.J. Behrens, M.D. Robson, C.E. Mackay, D.J. Higham, H. Johansen-Berg, Connectivity-based parcellation of human cortex using diffusion MRI: Establishing reproducibility, validity and observer independence in BA 44/45 and SMA/pre-SMA., Neuroimage 34 (2007) 204-11.

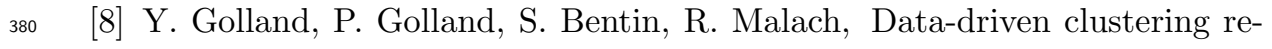
veals a fundamental subdivision of the human cortex into two global systems, Neuropsychologia 46 (2008) 540-53.

[9] L. Nanetti, L. Cerliani, V. Gazzola, R. Renken, C. Keysers, Group analyses of connectivity-based cortical parcellation using repeated k-means clustering, Neuroimage 47 (2009) 1666-1677.

[10] D. Meunier, R. Lambiotte, E.T. Bullmore, Modular and hierarchically modular organization of brain networks, Front. Neurosci. 4 (2010) 200.

[11] Y. Wang, T.Q. Li, Analysis of whole-brain resting-state fMRI data using hierarchical clustering approach, PLoS One 8 (2013).

[12] N. Slonim, G.S. Atwal, G. Tkacik, W. Bialek, Information-based clustering, Proc. Natl. Acad. Sci. U. S. A. 102 (2005) 18297-18302.

[13] N. Gorbach, Hierarchical information-based clustering for connectivitybased cortex parcellation, Front. Neuroinform. 5 (2011) 1-13.

[14] D. Moreno-Dominguez, A. Anwander, T.R. Knösche, A hierarchical method for whole-brain connectivity-based parcellation, Hum. Brain Mapp. 35 (2014) 5000-5025. 
[15] B. Thirion, G. Varoquaux, E. Dohmatob, J.B. Poline, Which fMRI clustering gives good brain parcellations?, Front. Neurosci. 8 (2014) 167.

[16] E. Bonmati, A. Bardera, I. Boada, M. Feixas, M. Sbert, Hierarchical clustering based on the information bottleneck method using a control process, Pattern Anal. Appl. 18 (2015) 619-637.

[17] T.M. Cover, J.A. Thomas, Elements of information theory, Wiley Series in Telecommunications, 1991.

[18] J.P. Crutchfield, N.H. Packard, Symbolic dynamics of noisy chaos, Phys. D: Nonlinear Phenomena 7 (1983) 201-223.

[19] D.P. Feldman, A brief introduction to: information theory, excess entropy and computational mechanics, 1997.

[20] N. Tishby, F. Pereira, W. Bialek, The information bottleneck method, in: Proc. 37th Annu. Allert. Conf. Commun. Control Comput., 1999, pp. 368-377.

[21] J. Burbea, C.R. Rao, On the convexity of some divergence measures based on entropy functions, IEEE Trans. Inf. Theory 28 (1982) 489-495.

[22] A. Strehl, J. Ghosh, C. Cardie, Cluster Ensembles - A Knowledge Reuse Framework for Combining Multiple Partitions, J. Mach. Learn. Res. 3 (2002) 583-617.

[23] C. Baldassano, D.M. Beck, L. Fei-Fei, Parcellating connectivity in spatial maps, PeerJ 3 (2015) 1-24.

[24] S. Achard, R. Salvador, B. Whitcher, J. Suckling, E.T. Bullmore, A resilient, low-frequency, small-world human brain functional network with highly connected association cortical hubs, J. Neurosci. 26 (2006) 63-72.

[25] P. Hagmann, M. Kurant, X. Gigandet, P. Thiran, V.J. Wedeen, R. Meuli, J.P. Thiran, Mapping Human Whole-Brain Structural Networks with Diffusion MRI, PLoS One 2 (2007). 
[26] Y. He, Z.J. Chen, A.C. Evans, Small-world anatomical networks in the human brain revealed by cortical thickness from MRI, Cereb. cortex 17 (2007) 2407-19.

[27] E. Bullmore, O. Sporns, O. Sporns, Complex brain networks: graph theoretical analysis of structural and functional systems, Nat Rev Neurosci 10 (2009) 186-198.

[28] O. Sporns, The human connectome: a complex network, Ann. N. Y. Acad. Sci. 1224 (2011) 109-125.

[29] M. Rubinov, O. Sporns, Complex network measures of brain connectivity: uses and interpretations, Neuroimage 52 (2010) 1059-1069.

[30] L. Cammoun, X. Gigandet, D.E. Meskaldji, J.P. Thiran, O. Sporns, K.Q. Do, P. Maeder, R. Meuli, P. Hagmann, Mapping the human connectome at multiple scales with diffusion spectrum MRI, J. Neurosci. Methods 203 (2012) 386-397.

[31] N. Tzourio-Mazoyer, B. Landeau, D. Papathanassiou, F. Crivello, O. Etard, N. Delcroix, B. Mazoyer, M. Joliot, Automated anatomical labeling of activations in SPM using a macroscopic anatomical parcellation of the MNI MRI single-subject brain, Neuroimage 15 (2002) 273-289.

[32] X. Zhao, Y. Liu, X. Wang, B. Liu, Q. Xi, Q. Guo, H. Jiang, T. Jiang, P. Wang, Disrupted small-world brain networks in moderate Alzheimer's disease: a resting-state fMRI study, PLoS One 7 (2012).

[33] M.C. Padula, M. Schaer, E. Scariati, M. Schneider, D. van de Ville, M. Debbané, S. Eliez, Structural and functional connectivity in the default mode network in 22q11.2 deletion syndrome, J. Neurodev. Disord. 7 (2015) 23.

[34] T. Simas, M. Chavez, P.R. Rodriguez, A. Diaz-Guilera, An algebraic topological method for multimodal brain networks comparisons, Front. Psychol. $6(2015) 904$. 
[35] M. Xia, J. Wang, Y. He, BrainNet Viewer: a network visualization tool for human brain connectomics., PLoS One 8 (2013). 